\title{
Long-term results of the open stent-grafting technique for extended aortic arch disease
}

\author{
Kazuo Shimamura, MD, ${ }^{a}$ Toru Kuratani, MD, PhD, ${ }^{a}$ Goro Matsumiya, MD, PhD, ${ }^{\text {a }}$ Masaaki Kato, MD, PhD, ${ }^{d}$ \\ Yukitoshi Shirakawa, MD, PhD, ${ }^{a}$ Hiroshi Takano, MD, PhD, ${ }^{\mathrm{c}}$ Noriyuki Ohta, MD, PhD, ${ }^{\mathrm{b}}$ and Yoshiki Sawa, MD, PhD ${ }^{\mathrm{a}}$
}

Objective: This report elucidates the long-term safety and effectiveness of extended aortic arch replacement with an open stent-grafting technique from our 12 years of experience.

Methods: From 1994 to 2004, 126 patients (mean age 67.8 years) with different pathologic conditions of the aortic arch with extension to the descending aorta (57 dissections [acute/chronic $=31 / 26$ ] and 69 aneurysms) were operated on with an open stent-grafting technique. During deep hypothermic circulatory arrest with selective cerebral perfusion, the stent graft was delivered through the transected proximal aortic arch, and arch replacement with a 4-branched prosthesis was performed.

Results: Operative mortality within 30 days was $3.2 \%$. Perioperative morbidity included $7(5.6 \%)$ strokes and $8(6.3 \%)$ spinal injuries (paraplegia in 3, transient paraparesis in 5). Sixty-three percent of the patients were extubated within 24 hours. In long-term follow-up (mean $60.4 \pm 36.5$ months, maximum 153 months), survival was $81.1 \%, 63.3 \%$, and $53.7 \%$ at 1,5 , and 8 years. Five $(3.9 \%)$ late endoleaks were observed but treated with successful additional endovascular repair. Freedom from endoleaks was $98.0 \%, 91.1 \%$, and $91.1 \%$ for 1,5 , and 8 years, respectively.

Conclusion: Long-term observation showed safety and good durability of the open stent-grafting technique for aortic arch disease. This technique could be an attractive treatment option for aortic arch aneurysm with distal extension and aortic dissection requiring aortic arch replacement.

From the Departments of Cardiovascular Surgery ${ }^{\mathrm{a}}$ and Anesthesiology, ${ }^{\mathrm{b}}$ Osaka University Graduate School of Medicine, the Department of Cardiovascular Surgery, ${ }^{\mathrm{c}}$ Osaka General Medical Center, and the Department of Cardiovascular Surgery, Morinomiya Hospital, Osaka, Japan.

Read at the Eighty-seventh Annual Meeting of The American Association for Thoracic Surgery, Washington, DC, May 5-9, 2007.

Received for publication May 3, 2007; revisions received Sept 27, 2007; accepted for publication Oct 31, 2007.

Address for reprints: Yoshiki Sawa, MD, $\mathrm{PhD}$, Department of Cardiovascular Surgery, Osaka University Graduate School of Medicine, 2-2 Yamadaoka, Suita, Osaka 565-0871, Japan (E-mail: sawa@surg1. med.osaka-u.ac.jp).

J Thorac Cardiovasc Surg 2008; 135:1261-9 $0022-5223 / \$ 34.00$

Copyright $\odot 2008$ by The American Association for Thoracic Surgery

doi:10.1016/j.jtcvs.2007.10.056
$\mathrm{D}$ irect surgical approach to pathologic conditions of the aortic arch has been a time-tested, established procedure with satisfactory results and durability. Median sternotomy is the preferred and most advantageous approach. However, performing anastomosis and hemostasis at the deep portion beyond the left subclavian artery is often associated with difficulties and also could induce recurrent laryngeal nerve injury. Moreover, when the distal end of the disease extends to the descending aorta, surgeons have to select extensive exposure of the distal aortic arch or left thoracotomy, which could raise the risk of respiratory complications.

Several approaches such as the clamshell incision, ${ }^{1}$ median sternotomy with left thoracotomy, ${ }^{2}$ and 2-stage repair with elephant trunk ${ }^{3,4}$ have been applied in this situation, but a less invasive procedure without left thoracotomy and with completion in a single stage is desired.

Meanwhile, as an emerging, less invasive alternative to conventional surgical repair, thoracic endovascular aortic repair (TEVAR) was brought into clinical use by Dake and his colleagues ${ }^{5}$ and reported in 1994. Our group has also been using TEVAR since 1993 after animal experimentation, ${ }^{6,7}$ and satisfactory early outcomes were achieved for type B aortic dissection. ${ }^{8}$ This method yielded a less invasive alternative for high-risk patients. However, applying TEVAR to aortic arch diseases was difficult because the aortic arch could not provide an adequate proximal landing zone. To mitigate the difficulties in both the distal anastomosis in conventional arch surgery and the proximal landing zone in TEVAR for the aortic arch, our group ${ }^{9}$ introduced 
Abbreviations and Acronyms

CSFD = cerebrospinal fluid drainage

CT = computed tomography

TEVAR $=$ thoracic endovascular aortic repair

Th = thoracic (vertebra)

the open stent-grafting technique in 1994. This technique, which subsequently has been described as frozen elephant trunk technique or stented elephant trunk in some articles, ${ }^{10-14}$ involves conventional replacement of the ascending aorta with a 4-branched graft and stent grafting into the descending aorta through the opened aortic arch. In 2002 , we ${ }^{15}$ reported that this technique is feasible for aortic dissection to obtain clot formation and shrinkage of the false lumen by closing the entry site in the aortic arch and providing continuous compression on the false lumen.

Previous reports ${ }^{10-15}$ described relatively small series, and it is still controversial whether this technique is safe and useful for long-term results.

The objective of this current report is to elucidate the feasibility of this technique for extended aortic arch replacement and to investigate the long-term durability and efficacy of this hybrid procedure from our 12 years of experience.

\section{Patients and Methods \\ Patients}

The open stent-grafting technique and the retrospective review of the records for publication were approved by the institutional review board. From February 1994 to July 2004, 126 patients having pathologic conditions of the aortic arch with extension to the descending aorta were operated on in a single center (Osaka General Medical Center, Osaka Prefectural Hospital) by an open stent-grafting technique. The selection for open stent-grafting technique was aortic arch aneurysms with extension to the descending aorta or aortic dissections that necessitated aortic arch replacement. These indications are listed as follows:

Aortic Arch/Proximal Descending Aortic Aneurysms

- Involving proximal portion of left subclavian artery and unable to be treated with TEVAR

- Extending distally to descending aorta and difficult/ unable to perform distal anastomosis in median approach

- Expected tight adhesion around distal anastomosis site (eg, redo operation)

Aortic Dissection

- Type A dissection with intimal tear in transverse arch or proximal descending aorta that could not be resected by hemiarch replacement

- Type A/type B dissection with aortic arch enlargement/ aneurysm

- Type B dissection with intimal tear in root of left subclavian artery (unable to close with TEVAR)

Informed consent was required in each case. Mean patient age was 67.8 years (range, 35-85 years), and 14 (11.1\%) patients were older than 80 years. Thirty-seven $(29.3 \%)$ operations were of
TABLE 1. Preoperative patients profiles

\begin{tabular}{lc}
\hline \multicolumn{1}{c}{ Demographic } \\
\hline Gender (M/F) & $86 / 40$ \\
Age (mean) & 67.8 \\
$>80$ y & 14 \\
Pathology & \\
Degenerative/atherosclerosis & 69 \\
Aortic dissection & 57 \\
Type A (acute/chronic) & $41(29 / 12)$ \\
Type B (acute/chronic) & $16(2 / 14)$ \\
Diameter of aneurysm (mm) & $63.6 \pm 10.6$ \\
Preoperative comorbidities (\%) & \\
Stroke & $21(16.7)$ \\
Coronary artery disease & $28(22.2)$ \\
Low LVEF (<40\%) & $5(4.0)$ \\
Chronic obstructive pulmonary disease & $10(7.9)$ \\
Renal failure (crn $>1.5$ mg/dL) & $17(13.5)$ \\
History of cancer & $9(7.1)$ \\
Previous surgery (\%) & \\
Cardiovascular & $6(4.8)$ \\
Descending aorta & $10(7.9)$ \\
Abdominal aorta & $18(14.3)$ \\
Preoperative status & \\
Emergency (\%) & $37(29.4)$ \\
Malperfusion with aortic dissection & \\
Cerebral artery & 3 \\
Coronary artery & 4 \\
$\quad$ Visceral artery & 3 \\
$\quad$ Lower extremity & 7 \\
\hline
\end{tabular}

$L V E F$, Left ventricular ejection fraction.

an emergency status. Preoperative comorbidities are shown in Table 1. Ten $(7.9 \%)$ patients had previous graft replacement of descending or thoracoabdominal aorta, and $18(14.3 \%)$ had a history of abdominal aortic repair. There were $3(2.4 \%)$ patients with Marfan syndrome.

Fifty-seven (46.8\%) patients had aortic dissection: among these patients, 29 (49.2\%) had acute type A dissections involving malperfusion of organs in 10 and loss of conscious in 3. Twelve (24.4\%) of the dissections were chronic type A dissections, including 5 redo cases with enlargement of patent false lumen of the aortic arch after ascending aorta replacement in the acute phase. Sixteen $(30.5 \%)$ cases were type B dissections, involving 2 acute complicated cases and 14 chronic type $\mathrm{B}$ dissections with enlargement of the false lumen (average size $50.0 \pm 7.7 \mathrm{~mm}$, range $41-70 \mathrm{~mm}$ ). Sixtynine $(53.2 \%)$ were degenerative/atherosclerotic aneurysms with 9 $(13.4 \%)$ instances of rupture. The average diameter of the aneurysm was $63.6 \pm 10.6 \mathrm{~mm}$. All these aneurysms had distal extension to the descending aorta, which required stent graft delivery to the level of the sixth thoracic vertebra (Th6) in $21.4 \%$, Th7 in $35.4 \%$, and distally to Th8 in $44.2 \%$.

\section{Description of the Device}

The stent graft was custom-made. It was composed of a Gianturco stent (William Cook Europe A/S, Bjaeverskov, Denmark) and a noncoated polyester fabric graft (WSL graft, Ube, Japan). The diameter of the polyester graft was selected with the measurement results of 
preoperative 3-dimensional computed tomography (CT). The diameter of the landing zone was calculated by tracing the intimal circumference, and a graft oversized by $10 \%$ to $20 \%$ was selected. These methods were described in detail in a previous study. ${ }^{9}$ Average diameter of the stent graft was $25.9 \pm 3.5 \mathrm{~mm}$ for dissections and $29.5 \pm 3.2 \mathrm{~mm}$ for degenerative aneurysms. We first used the 30F sheath and pushing rod to deliver the stent graft into the descending aorta. Although this method did not fail to deploy the stent graft, inserting the rigid sheath into the descending aorta under the guidance of transesophageal echocardiography is not easy. In 2001 we developed a sheathless delivery method. The stent graft was mounted on a balloon catheter (20F silicone nephrostomy balloon catheter) and the stented portion was restrained by a silk string. After the stent graft had been inserted into the descending aorta through the transected aortic arch, withdrawal of the restraining string released the self-expandable stented part of the graft.

\section{Surgery}

The details of the operation have been described in previous reports. ${ }^{9,15}$ Via a median sternotomy, the ascending aorta, brachiocephalic artery, and left common carotid artery were dissected. The arterial return cannula was placed in the right axillary artery and femoral artery. Cardiopulmonary bypass was initially started with right axillary perfusion. During core cooling to a $24^{\circ} \mathrm{C}$ bladder temperature, reconstruction of the left subclavian artery, left common carotid artery, and proximal anastomosis of the 4-branched graft was performed (Figure 1, $A$ ). The left subclavian artery was reconstructed in an extramediastinal fashion at the left infra subclavian space, with the graft branch passing through the second intercostal space. The proximal end of the 4-branched graft was anastomosed to the ascending aorta after cardiac arrest was achieved by crossclamping the ascending aorta and antegrade administration of cardioplegic solution. Selective cerebral perfusion via the 4-branched graft and right axillary artery was established and the perfusion to the lower body was discontinued. The aortic arch was transected at a predetermined line between the brachiocephalic artery and left common carotid artery, and the stented portion of the stent graft was inserted to the descending aorta (Figure 1,B). After deployment, the stented portion was dilated with a balloon catheter under the guidance of transesophageal echocardiography to confirm that the stent graft was fully opened and not kinked. The nonstented graft portion was sutured to the transected stump of the aortic arch, and subsequently continuous anastomosis to the 4-branched graft was made in end-to-end fashion. The debris and air were carefully flushed out from the descending aorta with femoral blood return before this anastomosis was completed. During rewarming, the brachiocephalic artery was reconstructed and the procedure was completed (Figure 1, C). When the ascending aorta was intact and replacement was unnecessary, a bifurcated graft was anastomosed to the ascending aorta instead of 4-branched graft replacement. Reconstruction of the left common carotid artery and left subclavian artery was performed in the same fashion. To prevent ischemic spinal injury, when the time of lower body circulatory arrest extended over 30 minutes, blood return to lower body was established from femoral artery with occluding the stent graft by balloon catheter.

\section{Spinal Protection}

From January 1997, cerebrospinal fluid drainage (CSFD) was performed before the operation in elective cases with a history of aortic repair in the thoracoabdominal or abdominal aorta. CSFD was administered preoperatively in $16(12.7 \%)$ cases. When the pathologic condition required stent graft delivery lower than the ninth thoracic vertebra, a short stent graft was delivered in the descending aorta without excluding the aneurysm, and additional endovascular grafting was performed the next day for complete exclusion (delayed exclusion technique). From 2001, to avoid spinal cord ischemia, we started to apply the delayed exclusion technique because of the following reasons:

1. Technical reason. A longer stent graft is hard to control under the guidance of transesophageal echocardiography, and

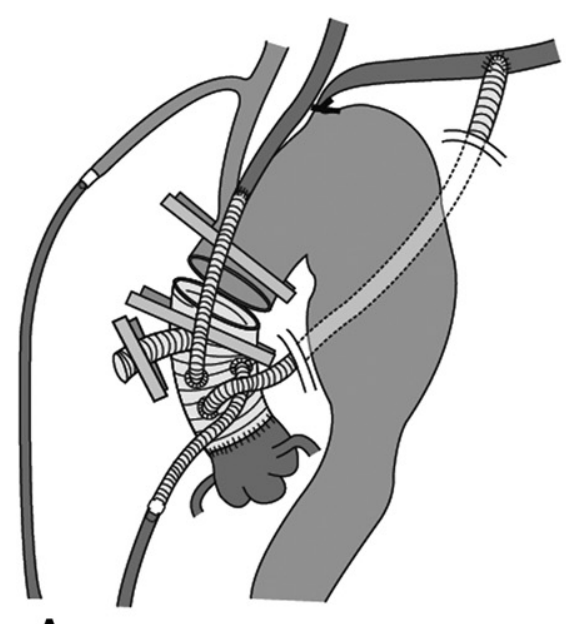

A

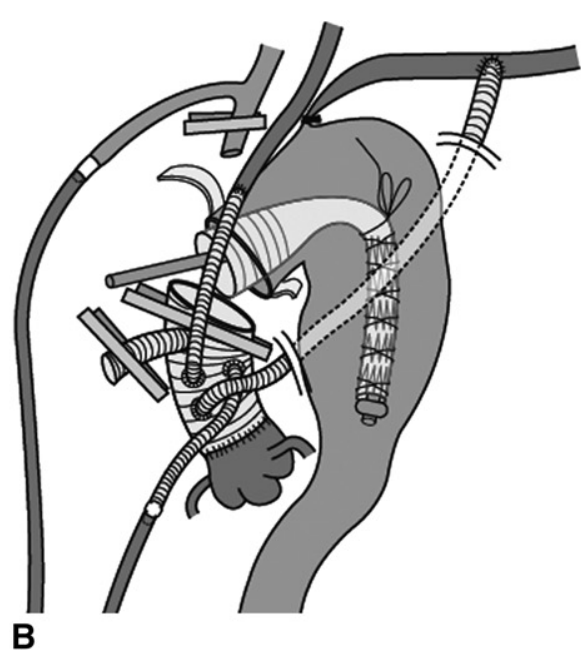

B

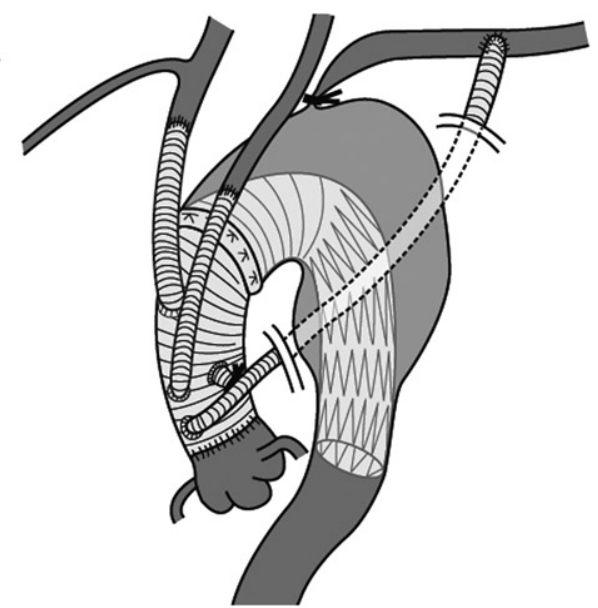

C

Figure 1. A, reconstruction of the left subclavian artery, left common carotid artery, and proximal anastomosis of the 4-branched graft. B, The aortic arch was transected at a predetermined line between the brachiocephalic artery and left common carotid artery, and the stent graft was inserted to the descending aorta. C, The brachiocephalic artery was reconstructed and the procedure was completed 
there is a risk of kinking. From our experience, an easily manageable length of the stent graft is less than $15 \mathrm{~cm}$, and this could reach around Th8 level.

2. Preservation of the spinal circulation. TEVAR provides excellent avoidance of spinal cord ischemia even when it covers the major intercostal artery connecting to the anterior spinal artery (Adamkiewicz artery). Delayed exclusion with additional endovascular grafting could maintain the spinal circulation, and we anticipate this technique could decrease the risk of spinal cord ischemia. Six (4.8\%) patients were treated with the delayed exclusion technique.

\section{Patient Follow-up}

The follow-up with contrast CT imaging was obtained before hospital discharge, 6 months after the operation, and yearly thereafter. A follow-up CT image was available for $81.9 \%$ of patients who survived longer than 6 months. Follow-up clinical status was obtained by medical records from an outpatient clinic or by contacting surviving patients or family members and primary care physician. Follow-up was $92.6 \%$ complete, averaging $60.4 \pm 36.5$ months (maximal follow-up 153 month, total cumulative follow-up $=533$ patient-years; 45 patient remaining at risk at 5 years).

\section{Statistical Analysis}

All data were reviewed retrospectively. Continuous variables are expressed as mean $\pm \mathrm{SD}$ and categorical variables as percentages. Survival and freedoms from aortic arch-related death, freedom from endoleaks, freedom from aortic intervention, and freedom from major adverse events were estimated by the Kaplan-Meier method.

\section{Results}

\section{Early Outcome}

The stent graft delivery was technically successful in all 126 patients. In $6(4.8 \%)$ patients kinking of the stent graft showed a pressure gradient greater than $20 \mathrm{~mm} \mathrm{Hg}$. All these patients were successfully treated intraoperatively by endovascular repair with additional bare stent deployment. Concomitant procedures included 4 aortic root replacements, 6 aortic valve replacements, and 8 coronary artery bypass grafts. There were 7 cases of type A acute aortic dissection with malperfusion of the lower extremity. To recanalized the malperfusion, we performed endovascular repair in 4 patients (3 iliac stenting and 1 stenting of the descending aorta) and bypass grafting in 3 ( 2 aortofemoral bypass and 1 femorofemoral bypass) intraoperatively. The average operative, cardiopulmonary bypass, and circulatory arrest times of the lower body were 401, 147, 31 minutes, respectively.

Operative mortality within 30 days was 4 of 126 (3.2\%). Two death were in acute type A dissection (1 due to postoperative heart failure, 1 due to uncontrollable hemmorage from the aortic root with coagulopathy), and another 2 death were in degenerative aneurysm ( 1 due to stroke, 1 due to postoperative thoracoabdominal aneurysm rupture). There were 7 inhospital deaths after 30 days (in-hospital mortality 5.5\%). The causes of in-hospital mortality were brain infarction $(n=1)$, sepsis $(n=3)$, aortoesophageal fistula $(n=1)$, rupture
TABLE 2. Causes of early and late death

\begin{tabular}{lccc}
\hline \multicolumn{1}{c}{ Causes of death } & Early $(<\mathbf{y}$ y) & Late $(>\mathbf{1}$ y) & Total \\
\hline Aortic & & & \\
$\quad$ Rupture (treatment failure) & 0 & 1 & 1 \\
Aortoesophageal fistula & 1 & 1 & 2 \\
Rupture of other aortic aneurysm & 3 & 1 & 4 \\
Nonaortic & & & \\
Cancer & 0 & 7 & 7 \\
Brain infarction & 3 & 2 & 5 \\
Subarachnoid hemorrhage & 1 & 1 & 2 \\
Pneumonia & 0 & 6 & 6 \\
Sepsis & 3 & 1 & 4 \\
Renal failure & 1 & 2 & 3 \\
Acute myocardial infarction & 1 & 1 & 2 \\
Arrhythmia & 0 & 1 & 1 \\
Ischemic colitis & 0 & 1 & 1 \\
Ileus & 0 & 1 & 1 \\
Gastric bleeding & 0 & 1 & 1 \\
Cirrhosis & 1 & 0 & 1 \\
Suicide & 1 & 0 & 1 \\
Sudden death & 1 & 0 & 1 \\
\hline
\end{tabular}

of thoracoabdominal aortic aneurysm $(\mathrm{n}=1)$, and acute myocardial infarction $(\mathrm{n}=1)$. Aortoesophageal fistula occurred in a patient with ruptured type $B$ aortic dissection with mediastinal hematoma. This patient was treated successfully with the open stent-grafting technique and the aneurysm was successfully excluded, but an aortoesophageal fistula developed after the mediastinal hematoma had absorbed at 38 days postoperatively. The causes of early death within 1 year are listed in Table 2. Perioperative morbidity included 7 (5.6\%) strokes, 3 (2.4\%) cases of paraplegia, and 5 (3.9\%) cases of transient paraparesis. No spinal cord ischemia was observed in the patients treated by the delayed exclusion technique. The average number of packed red blood cells transfusions was $14.0 \pm 3.1$ units and re-exploration for bleeding was required in $3(2.4 \%)$ patients. In $6(4.8 \%)$ with renal failure, transient hemodialysis was required. The length of stay in the intensive care unit ranged from 0 to 60 days, median 4 days.

Postoperative hoarseness occurred in 12 (9.5\%) patients; however, 7 (58\%) of 12 were fully recovered before hospital discharge. Sixty-three percent of the patients were extubated within 24 hours, and $8(6.3 \%)$ required tracheostomy.

The demographics of patients with paraplegia and transient paraparesis are shown in Table 3. All patients with transient paraparesis had recovered enough to be able to walk by themselves. The median hospital stay was 29 days, and $11(8.7 \%)$ of the patients were discharged to an institution for rehabilitation.

\section{Late Outcome}

Survival. Actuarial survival estimates 1,5 , and 8 years after the procedure were $81.1 \%, 63.3 \%$, and $53.7 \%$, respectively (Figure 2). Causes of deaths are listed in Table 2. There 
TABLE 3. Demographics of spinal cord ischemia

\begin{tabular}{|c|c|c|c|c|}
\hline Spinal cord ischemia & Pathology & Distal landing zone & Risk factor & Comment \\
\hline Paraplegia & TAA & Th8 & AAA repair & \\
\hline Paraplegia & $\mathrm{AD}(\mathrm{A})$ & Th6 & Hypotension & Stent graft stenosis \\
\hline Parapleiga & $A D(A)$ & Th6 & Hypotension & Stent graft stenosis \\
\hline Transient paraparesis & TAA & Th9 & AAA repair & \\
\hline Transient paraparesis & TAA & Th8 & AAA repair & \\
\hline Transient paraparesis & TAA & Th8 & & \\
\hline Transient paraparesis & $A D(A)$ & Th6 & Hypotension & $\begin{array}{l}\text { Preoperative } \\
\text { malperfusion }\end{array}$ \\
\hline Transient paraparesis & $A D(A)$ & Th6 & Hypotension & $\begin{array}{l}\text { Preoperative } \\
\text { malperfusion }\end{array}$ \\
\hline
\end{tabular}

$T A A$, Degenerative/atherosclerotic aneurysm; $A D(A)$, aortic dissection (type $\mathrm{A}$ ); $A A A$. abdominal aortic aneurysm.

was $1(0.79 \%)$ late death resulting from rupture of a treated aortic arch aneurysm that was caused by leaking from the proximal suture site of the stent graft. One $(0.8 \%)$ late fatal aortoesophageal fistula had occurred. This case involved a huge degenerative aneurysm encompassing the whole thoracic aorta with chest pain and a hematoma around the aneurysm (sealed rupture). This patient was treated successfully by extended aortic arch replacement with the open stentgrafting technique; the stent graft was delivered to the 11th thoracic vertebra level by a delayed exclusion technique. The patient had an uneventful recovery, and CT 12 months after the procedure showed shrinkage of the aneurysm (70 $\mathrm{mm}$ to $61 \mathrm{~mm}$ ). However, 28 months after the procedure, CT showed type III endoleak. Additional TEVAR was planned, but the aorta ruptured before treatment was instituted. Emergency TEVAR could have saved the patient's life, but fatal aortoesophageal fistula had occurred.

Brain infarctions as the cause of death within 1 year were promoted by the surgery, unfortunately. Two late brain infarctions were observed, but neither of them resulted from cerebral graft occlusion or graft thrombosis. Rupture of other aortic aneurysms had occurred while one of the patients was waiting for the next operation; the other patient had refused further treatment. All these patients showed complete exclusion of the treated arch aneurysm on postoperative CT scans, and none of these were associated with stent graft dislodgment or perforation.

Operative mortality, aortoesophageal fistula, stent graftrelated mortality (perforation, occlusion, and thromboembolism), and cerebral graft-related mortality were defined as aortic arch-related death, and the freedom from aortic arch-related death was $95.8 \%, 92.4 \%$, and $92.4 \%$ at 1,5 , and 8 years, respectively (Figure 3 ).

Endoleaks and aortic intervention. Follow-up CT was obtained in $81.9 \%$ of patients who survived longer than 6 months. In degenerative aneurysms, $68.3 \%$ of the aneurysms showed disappearance or shrinkage in late followup. In aortic dissections, $78.6 \%$ have achieved shrinkage of

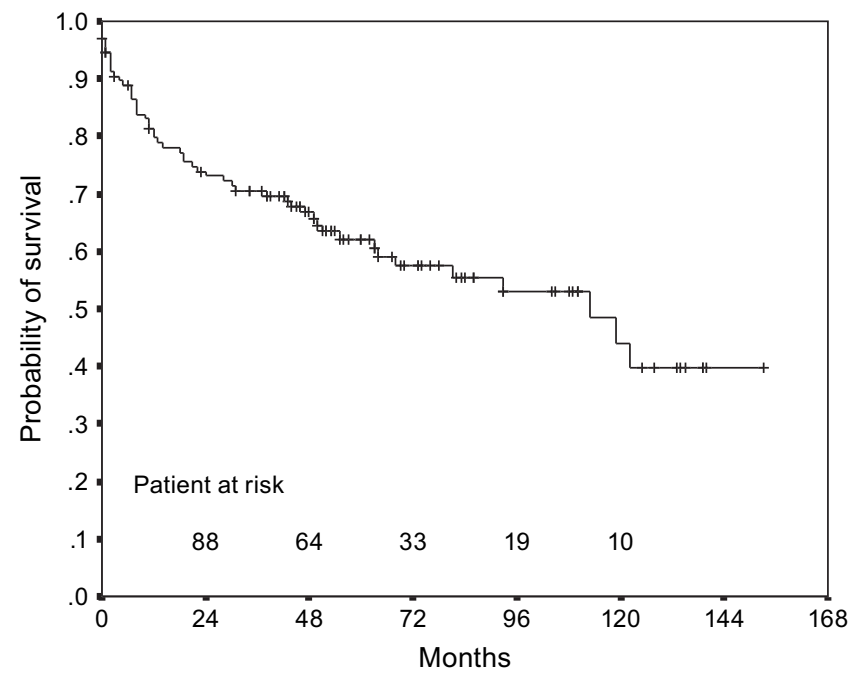

Figure 2. Overall survival of all 126 patients.

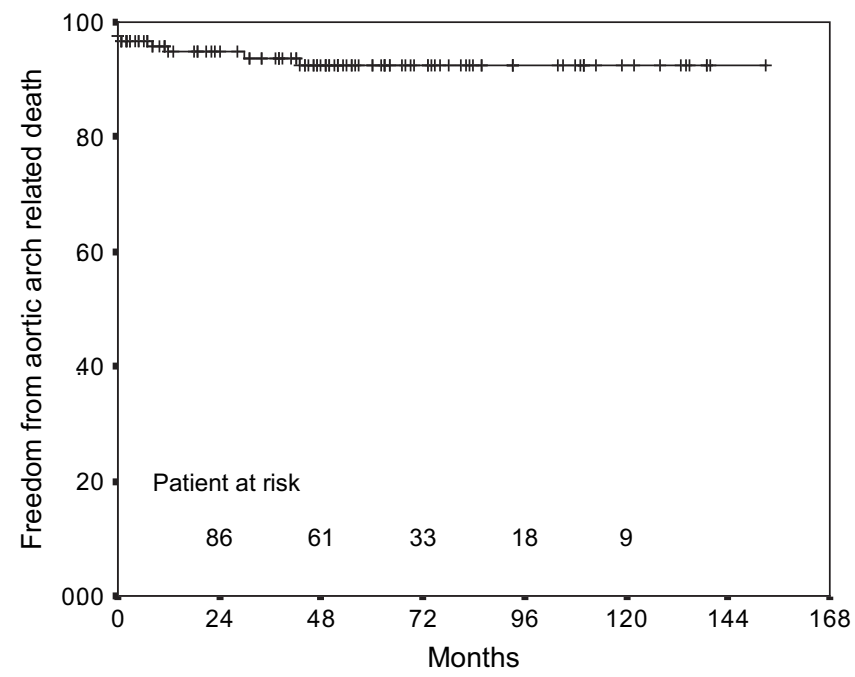

Figure 3. Freedom from aortic arch-related death. 
the false lumen. Among these patients, 30.3\% showed complete disappearance of the false lumen in the entire aorta, and $48.4 \%$ showed disappearance of the false lumen in the thoracic lesion. Creation of a new intimal tear by the edge of the stent graft was not observed in any case.

Late endoleaks were observed in $5(3.9 \%)$ cases (4 in degenerative/atherosclerotic aneurysms, 1 in chronic type B aortic dissection). Type I endoleak from the distal end of the stent graft had occurred in 3 cases, and type III endoleak from the stent-in-stent site in 2 cases. Endoleaks were observed an average of 24.8 months postoperatively, and all of them were successfully treated with additional endovascular repair. Actuarial freedom from endoleaks was $98.0 \%$, $91.1 \%$, and $91.1 \%$ for 1 year, 5 years, and 8 years, respectively.

Intervention of another aortic lesion was administered in $10(7.9 \%)$ cases. There were 7 TEVARs and 3 graft replacements ( 1 thoracoabdominal aortic aneurysm and 2 aascending aortic aneurysms). All of these cases that required intervention for other aortic lesions were aortic dissection cases. The average postoperative duration for intervention of other aortic lesions was 38.3 months (range 3-89 months). Actuarial freedom from aortic intervention (freedom from intervention of endoleaks and other aortic aneurysms) was $97.2 \%, 83.8 \%$, and $75.4 \%$ for 1,5 , and 8 years, respectively.

Major adverse events. In-hospital death, stroke, spinal cord ischemia, aortic rupture, aortoesophageal fistula, and endoleak were defined as major adverse events, and the freedom from major adverse events was $79.9 \%, 73.8 \%$, and $73.8 \%$ at 1,5 , and 8 years, respectively.

\section{Discussion}

The open stent-graft technique is a hybrid procedure that combines conventional aortic surgery and endovascular repair. By using the stent graft as an alternative to the distal anastomosis, surgeons can avoid extended dissection of the distal arch and left thoracotomy. This procedure could be completed by making the distal aortic incision between the brachiocephalic artery and left common carotid artery, which provides a better surgical view and hemostasis than traditional anastomosis in aortic arch replacement. Insertion of the stent graft could be performed in a few minutes. Thus this procedure has an advantage in arch aneurysms extending distally to the descending aorta, it being difficult or impossible to perform distal anastomosis by the median approach, or in aneurysms that are expected to have tight adhesion around the distal anastomosis site (eg, redo cases). It is also useful for type A dissections with an intimal tear in the transverse arch or proximal descending aorta that cannot be resected by hemiarch replacement.

In this cohort of 126 patients, the overall operative mortality within 30 days was $3.2 \%$. In degenerative aneurysms, the operative mortality within 30 days was $2.9 \%$. This is comparable with the operative mortality within 30 days of the first- stage operation with the elephant trunk technique in contemporary reports $(2.1 \%-12 \%) .{ }^{16,17}$ However, the elephant trunk technique requires a second-stage operation (mortality $4 \%$ $8.5 \%$ ) to complete the procedure, and there are even a considerable number of patients who do not survive to the second operation. The open stent-grafting technique has an advantage over the elephant trunk technique in that it completes the procedure within a single operation.

In acute type A dissection, the entire intimal tear resection is desirable to achieve a good fate of the remaining dissected aorta. ${ }^{18}$ However, extending the procedure to the aortic arch could be associated with increasing mortality. ${ }^{18}$ In our series, the mortality in acute dissection is satisfactorily low (6.9\%), and this could relate to the fact that this procedure could achieve total arch repair without manipulating more distally to the left common carotid artery, which facilitates the control of bleeding.

Postoperative occurrence of vocal cord paralysis after aortic surgery is considered to be an independent predictor of pulmonary complications and leads to a longer duration of hospital stay. ${ }^{19}$ Even with preservation of the recurrent laryngeal nerve, vocal cord paralysis occurs in $21.9 \%$ to $32 \%$ of aortic arch operations and in most cases does not return to normal movement. ${ }^{20}$ Our procedure does not require resection distally to the left artery, and it has the advantage of avoiding recurrent nerve injury. Postoperative vocal cord paralysis was observed in $9.5 \%$, and $58 \%$ of them had fully recovered during hospitalization. Mechanical ventilation for more than 72 hours was required in $20.0 \%$ in our series and could be low in comparison with other series of aortic arch replacement through a left thoracotomy (36-50\%). ${ }^{21,22}$ These results suggest that the open stent-grafting technique could contribute to reduced postoperative respiratory failure.

Spinal cord ischemia is a devastating complication of aortic surgery. Our series showed $3(2.4 \%)$ cases of paraplegia and $5(3.9 \%)$ of paraparesis. Among the 3 patients with paraplegia, 2 had intraoperatively hypotensive perfusion of the lower body associated with stent graft stenosis. Inasmuch as hypotension is widely known to be a risk factor for the development of spinal cord ischemia, ${ }^{23}$ stent graft stenosis is an important pitfall of this procedure. Designing the stent graft carefully is mandatory when custom-made stent grafts are used.

Among the 5 cases of temporal paraparesis, 2 were type A acute dissections with preoperative malperfusion. Both of them had a clotted false chamber and severely narrowed true chamber in the descending aorta associated with hypoperfusion of the visceral arteries and lower extremity, which raised concern about the occurrence of spinal cord ischemia. The remaining 3 cases except for the hypoperfusion cases were associated with a history of abdominal aortic repair. From 1997, we started to administer CSFD before the operation in cases of previous thoracoabdominal or abdominal 
aortic repair. No spinal cord ischemia was observed since then except the 2 cases of acute type A dissection with preoperative malperfusion.

In our study, survival at 1,5 , and 8 years was $81.1 \%$, $63.3 \%$, and $53.7 \%$, respectively. Long-term results of extended aortic arch repair are not well described, but our result is comparable with those of other series of simple aortic arch replacement (5-year survival 46\%-79\%). ${ }^{23-25}$

Despite encouraging early results, there are few reports describing long-term results of endovascular stent graft repair. Demers and his colleagues ${ }^{26}$ reported in their study of 103 TEVARs for descending thoracic aorta that freedom from endoleak was $78 \%, 64 \%$, and $50 \%$ at 1,5 , and 8 years, respectively. Freedom from endoleak in this series was $98.0 \%, 91.1 \%$, and $91.1 \%$ for 1,5 , and 8 years, respectively, which could be superior to TEVAR. TEVAR is inevitably associated with some frequency of proximal type I endoleak in the early and long-term follow-up periods, but this is avoidable in the open stent-grafting technique because the proximal end of the stent graft was sutured in a surgical fashion. All 5 patients who had a late distal endoleak were successfully treated with additional TEVAR, and there have been no secondary endoleaks.

Although shrinkage of the aneurysm sac after endovascular repair may be desirable, the persistence of the aneurysm does not represent a failure because a stable aneurysm without endoleak has not been linked to any untoward effects. ${ }^{27}$

On the other hand, sac enlargement implies increased pressure within the aneurysm sac and should be treated promptly. In our series, 3 aneurysms showed enlargement on late follow-up and all of them were associated with type I/type III endoleaks. Also, we have to consider that the intercostal arteries are opened to the excluded aneurysm (type II endoleak) and this requires that the transected stump of the aortic arch be sutured to the stent graft. Inasmuch as these issues should be carefully studied, we perform postoperative CT with contrast medium at the arterial phase and delayed phase to detect endoleaks.

However, type I/type III endoleaks can be treated with additional endovascular repair with a good result. According to type II endoleak, Ohki and colleagues ${ }^{28}$ had reported a study using an implantable wireless aneurysm pressure sensor for endovascular repair of abdominal aorta. They showed that the postexclusion pulse pressure was significantly decreasing. In no patient in our series was trouble caused by the pressure inside the aneurysm, such as continuous leaking from ruptured aneurysm or enlargement of the aneurysm with type II endoleak. From these results, the pressure inside the aneurysm (endotension) could be low enough in the clinical point of view.

\section{Limitation of the Study}

This is a retrospective cohort study, and the lack of a concurrent control group restricts direct comparison with total arch replacement associated with left thoracotomy. For the precise advantage of open stent-grafting technique to be elucidated, a prospective case-control study would be required. This study includes our earliest experience with stent grafting, so both the technical immaturity and the lack of adequate spinal protection could have caused spinal ischemia. Improved stent graft design, delivery system, and consideration of spinal protection could be associated with better outcomes.

\section{Conclusion}

In this study of 126 patients treated with an open stentgrafting technique, satisfactory early results were obtained in both aortic dissections and aortic arch aneurysms with distal extension to the descending aorta. Regarding long-term outcomes, acceptable survival and good avoidance of aortic arch-related death was obtained. The incidence of endoleaks was satisfactorily low and the patients could be rescued by additional endovascular repair. In aortic dissection, this procedure could obtain good clot formation and shrinkage of the false lumen. These results suggest that the open stent-grafting technique is a safe and effective procedure and might be a good alternative to conventional prosthetic replacement for aortic dissections and arch aneurysm with distal extension to the descending aorta.

\section{References}

1. Rokkas CK, Kouchoukos NT. Single stage extensive replacement of the thoracic aorta: the arch-first technique. J Thorac Cardiovasc Surg. 1999; 117:99-105.

2. Massimo CG, Perna AM, Cruz Quadron EA, Artounian RV. Extended and total simultaneous aortic replacement: latest technical modifications and improved results with thirty-four patients. J Card Surg. 1997;12: 261-9.

3. Borst HG, Walterbusch G, Schaps D. Extensive aortic replacement using "elephant trunk" prosthesis. Thorac Cardiovasc Surg. 1983;31:37-40.

4. Crawford ES, Coselli JS, Svensson LG, Safi HJ, Hess KR. Diffuse aneurysmal disease (chronic aortic dissection, Marfan, and mega aorta syndromes) and multiple aneurysm. Treatment by subtotal and total aortic replacement emphasizing the elephant trunk operation. Ann Surg. 1990;211:521-37.

5. Dake MD, Miller DC, Semba CP, Mitchell RS, Walker PJ, Liddell RP. Transluminal placement of endovascular stent-grafts for the treatment of descending thoracic aneurysms. N Engl J Med. 1994;331:1729-34.

6. Kato M, Ohnishi K, Kaneko M, Imagawa H, Ueda T, Kuratani T, et al. Development of an expandable intra-aortic prosthesis for experimental aortic dissection. ASAIO J. 1993;39:M758-61.

7. Kato M, Matsuda T, Kaneko M, Ueda T, Kuratani T, Yoshioka Y, et al. Experimental assessment of newly devised transcatheter stent-graft for aortic dissection. Ann Thorac Surg. 1995;59:908-15.

8. Kato M, Matsuda T, Kaneko M, Kuratani T, Mizushima T, Seo Y, et al. Outcomes of stent-graft treatment of false lumen in aortic dissection. Circulation. 1998;98(Suppl):II305-12.

9. Kato M, Ohnishi K, Kaneko M, Ueda T, Kishi D, Mizushima T, et al. New graft-implanting method for thoracic aortic aneurysm of dissection with a stented graft. Circulation. 1996;94(Suppl II):II188-93.

10. Flores J, Kunihara T, Shiiya N, Yoshimoto K, Matsuzaki K, Yasuda K. Extensive deployment of the stented elephant trunk is associated with an increased risk of spinal cord injury. J Thorac Cardiovasc Surg. 2006; 131:336-42.

11. Usui A, Fujimoto K, Ishiguchi T, Yoshikawa M, Akita T, Ueda Y. Cerebrospinal dysfunction after endovascular stent-grafting via a median 
sternotomy: the frozen elephant trunk procedure. Ann Thorac Surg. 2002;74:S1821-4.

12. Baraki H, Hagl C, Khaladj N, Kallenbach K, Weidemann J, Haverich A, et al. The frozen elephant trunk technique for treatment of thoracic aortic aneurysms. Ann Thorac Surg. 2007;S819-23.

13. Liu ZG, Sun LZ, Chang Q, Zhu JM, Dong C, Yu CT, et al. Should the 'elephant trunk' be skeletonized? Total arch replacement combined with stented elephant trunk implantation for Stanford type A aortic dissection. J Thorac Cardiovasc Surg. 2006;131:107-13.

14. Ishihara H, Uchida N, Yamasaki C, Sakashita M, Kanou M. Extensive primary repair of the thoracic aorta in Stanford type A acute dissection by means of a synthetic vascular graft with a self-expandable stent. J Thorac Cardiovasc Surg. 2002;123:1035-40.

15. Kato M, Kuratani T, Kaneko M, Kyo S, Ohnishi K. The results of total arch graft implantation with open stent-graft placement for type A aortic dissection. J Thorac Cardiovasc Surg. 2002;124:531-40.

16. Svensson LG, Kim KH, Blackstone EH, Alster JM, McCarthy PM, Greeenberg RK, et al. Elephant trunk procedure: newer indications and uses. Ann Thorac Surg. 2004;78:109-16.

17. LeMaire SA, Carter SA, Coselli JS. The elephant trunk technique for staged repair of complex aneurysms of the entire thoracic aorta. Ann Thorac Surg. 2006;81:1561-9.

18. Crawford ES, Kirklin JW, Naftel DC, Svensson LG, Coselli JS, Safi HJ. Surgery for acute dissection of ascending aortic dissection: should the arch be included? J Thorac Cardiovasc Surg. 1992;104:46-59.

19. Ohta N, Kuratani T, Hagihira S, Kazumi K, Kaneko M, Mori T. Vocal cord paralysis after aortic surgery: predictors and clinical outcome. $J$ Vasc Surg. 2006;43:721-8.

20. Ishimoto S, Ito K, Toyama M, Kawase I, Kondo K, Oshima K, et al. Vocal cord paralysis after surgery for thoracic aortic aneurysm. Chest. 2002;121:1911-5.

21. Kouchoukos NT, Mauney MC, Masetti P, Castner CF. Optimization of aortic arch replacement with a one-stage approach. Ann Thorac Surg. 2007;83:S811-4.

22. Tominaga R, Kurisu K, Ochiai Y, Nakashima A, Masuda M, Morita S, et al. Total arch replacement through the L-incision approach. Ann Thorac Surg. 2003;75:121-5.

23. Crawford ES, Svensson LG, Hess KR, Shenaq SS, Coselli JS, Safi HJ, et al. A prospective randomized study of cerebrospinal fluid drainage to prevent paraplegia after high-risk surgery on the thoracoabdominal aorta. J Vasc Surg. 1991;13:36-45.

24. Okita Y, Ando M, Minatoya K, Kitamura S, Takamoto S, Nakajima N. Predictive factors for mortality and cerebral complications in arteriosclerotic aneurysm of aortic arch. Ann Thorac Surg. 1999;67:72-8.

25. Kazui T, Washiyama N, Muhammad BA, Terada H, Yamashita K, Takinami M, et al. Total arch replacement using aortic arch branched grafts with the aid of antegrade selective cerebral perfusion. Ann Thorac Surg. 2000;70:3-8; discussion 8-9.

26. Demers P, Miller DC, Mitchell RS, Kee ST, Sze D, Razavi MK, et al. Midterm results of endovascular repair of descending thoracic aortic aneurysms with first-generation stent grafts. J Thorac Cardiovasc Surg. 2004;127:664-73.

27. Cho JS, Dillavou ED, Rhee RY, Makaroun MS. Late abdominal aortic aneurysm enlargement after endovascular repair with the Excluder device. J Vasc Surg. 2004;39:1236-42.

28. Ohki T, Ouriel K, Silveria PG, Katzen B, White R, Criado F. Initial results of wireless pressure sensing for endovascular aneurysm repair: the APEX trial-acute pressure measurement to confirm aneurysm sac exclusion. J Vasc Surg. 2007;45:236-42.

\section{Discussion}

Dr John Elefteriades (New Haven, Conn). I congratulate the authors on an outstanding paper on hybrid therapy for aortic arch aneurysm. The number of patients is large, the follow-up is quite complete, and the duration of follow-up is long. The mortality and stroke rates are excellent. I do take issue with the underlying theme of the paper, that a direct surgical approach to the aortic arch is to be feared and avoided in favor of extra-anatomic and stent-graft therapy. Direct approaches to the aortic arch are safe, durable, and time tested. Many series, including our own recently presented experience, show stroke and mortality rates with the conventional approach that are very comparable with those in your presentation.

Also, our experience shows that the descending aorta is not commonly problematic, even in the long term, after successful type A repair, raising doubt about whether adjunctive stent therapy in the descending aorta is really necessary.

I have 3 questions. My first question has to do with early mortality. You list your hospital mortality as 3\%, but by 6 months $19 \%$ of the patients are dead. How did another $16 \%$ of the patients die within 6 months of hospital discharge? What was going on clinically and radiographically with their aortas and their brains before death?

My second question has to do with anatomic morphology and selection for operation. It appears that a large proportion of cases in this paper are not really extended arch lesions, as the title indicates, but rather typical type A and type B dissections. I am uncomfortable with the classification of the large number of patients with chronic type B dissection in this series as having "arch" lesions. Descending dissections are nearly always limited to the region distal to the subclavian artery. These lesions are well treated by a relatively straightforward resection via left thoracotomy on left atrial-femoral artery bypass. With a clamp placed between the left carotid and left subclavian arteries, one has excellent exposure for the proximal anastomosis. Why did the authors classify these as arch lesions and perform their complex extra-anatomic repair?

For the third question, let us consider the failures of therapy. How do you explain the persistence or progressive enlargement of the aneurysm in $25 \%$ of your patients?

Congratulations on an excellent presentation.

Dr Shimamura. Thank you, Dr Elefteriades, for your kind comments and important questions.

Regarding the first question about mortality, the early mortality included 3 patients with postoperative strokes, and we lost these 3 patients within 1 year. It could be said that we have lowered the quality of life of the patients postoperatively, but the death is not associated directly to the stent graft. Also, there is a patient who had an fatal aortoesophageal fistula, as I showed in my slides. We lost this patient after successful treatment for a ruptured aneurysm however, this endoluminal treatment does not resect the whole aneurysm, so this kind of problem needs more investigation. Most of the late deaths are associated with the relatively high risk of the background of the patients. We introduced stent-graft treatment in clinical use in 1993, and this is the very first in Japan. Many patients with severe comorbidities were sent to our institution, and we have to deal with these high-risk patients. This could explain the relatively poor overall survival in the long term. However, we believe that avoidance of the aortic arch-related deaths is satisfactory.

Your second question concerned the indication for this procedure. Yes, we include type B dissection for this procedure, but the indication is only for the complication-specific treatment for type $\mathrm{B}$ dissection. We undertook this procedure for the patient with type B dissection who has a very proximal intimal tear near to the subclavian artery. These tears cannot be treated with endovascular repair. Of course, there are options to do graft replacement with a left thoracotomy, but we think that closure of the intimal tear via a median approach is compatible with this treatment, and the results are satisfactory in our study. 
Regarding the third question, the majority of the aneurysms have an extension distally, and it was thought to be difficult to treat that extension with conventional treatment via a median sternotomy. Our series contains about $20 \%$ of patients who have a relatively proximal location of the distal end of the aneurysms, and these could be treated with traditional surgical repair. However, this procedure has an advantage even in these aneurysms, because with the stent graft you can alternate your distal anastomosis and manipulate only in the proximal arch. This could be a very easy way to control the bleeding.

Dr Bruce W. Lytle (Cleveland, Ohio). Regardless of the specific indications for how often you do a debranching or whether this is just an extension of a conventional arch repair, the concept of putting in a stent graft during an open aortic operation, perhaps to extend it to the descending aorta, makes a lot of sense. We certainly have tried to do this. One of the things I have been really disappointed in is the lack of response of our industrial partners to this issue. We have tried to get a number of the companies interested in making devices specifically for the purpose of being put in at the time of surgery. They just cannot seem to get this.

The way you wrap the string around the graft is very clever, but are you still doing it that way after 14 years? Has there not been some company that has agreed to manufacture that on sort of a prospective basis?

Dr Shimamura. We do not use the sheath to deliver the stent graft, but we have no company that makes this kind of commercially available stent graft. I hear that that kind of device is available in Germany, and I think the production of such a device could expand this technique widely.

Dr Lytle. I invite our colleagues to help our industrial partners to understand that we are interested in this, because regardless of the specific indication, there is no doubt that this concept will have some degree of usefulness. 